\title{
AN ADAPTIVE ALGORITHM BASED AUTHENTICATION SYSTEM FOR REAL-TIME BIOMETRIC TIME-ATTENDANCE SYSTEM
}

\author{
Thejaswini.P', Anu H' ${ }^{\mathbf{1}}$, D. Mahesh Kumar ${ }^{2}$, Aravinda H $\mathbf{S}^{\mathbf{1}}$. \\ ${ }^{1}$ Department of ECE, JSS Academy of Technical Education, Bangalore. \\ ${ }^{2}$ Department of E\&IE, JSS Academy of Technical Education, Bangalore.
}

\begin{abstract}
Biometric features are physical and biological characteristics that are unique to a person and can be used to accomplish authentication based on the particular modality. The main purpose of implementation of an Adaptive autocorrection technique for biometric timeattendance system is to improve the matching rate of fingerprint verification under the condition when fingerprint patterns vary due to environmental parameter like temperature. An Adaptive auto-correction technique is proposed which auto-corrects the reference fingerprint template at the time of genuine user rejection. The proposed technique is implemented on commercially available biometric device which uses Innovatrics, a standard commercially available extractor and matcher. Evaluation is carried out on 250 fingerprint templates of 10-users captured at varying temperature from $25^{\circ} \mathrm{C}$ to $0^{\circ} \mathrm{C}$. The experimental analysis will be carried out to improve the recognition rate.
\end{abstract}

Keywords: Biometric, minutiae, Innovatrics, template, Adaptive, autocorrection, matching score

\section{Introduction}

Automatic identification of individuals based on their physical and/or behavioral characteristics is termed as Biometrics. Advancement in the technology has led biometrics to replace the conventional access control and time attendance systems [10]. These biometric traits are very unique features of humans that remain stable throughout the life span under normal conditions [1]. Indeed, it's observed that this unique fingerprint pattern differs as a result of environmental variations like temperature, humidity, dust and also due to ageing of the person [11].

Biometric devices play vital role in identification of genuine user/subject. It is necessary for the devices to be robust to avoid fraudulent. Principle behind fingerprint based biometric system is comparison of live fingerprint image with the stored reference fingerprint image to find the matching [1]. Any variations in the captured fingerprint image will affect the quality leading to poor matching with the stored reference fingerprint image which in turn fails to recognize the same person's identity [4][10] [12], leading to the false rejection of the genuine user/subject. [3].

Commercially available biometric devices exhibit varied performance due to seasonal variations with the variation of environmental parameters like temperature. These variations lead to poor fingerprint matching which in turn fails to match same user fingerprint with the reference fingerprint template stored in the database. This calls for reregistration of fingerprint many times to match with the reference fingerprint due 
to temperature variation. As the temperature decreases, the failure rate increases due to variation in fingerprint pattern which are quite sensitive to temperature. Some of the popular commercially available fingerprint-based extractor and matching algorithm are Suprema [21], Morpho [22], Innovatrics [23], Mega matcher [24], secugen [25] etc.

\section{Related Work}

Jacqueline A. Speir [5] proposed a frequency filtering algorithm to enhance the fingerprint image quality. To quantify the impact of proposed algorithm, two database of same fingerprint images were created, one with high quality fingerprint image and other with noise or low quality. The proposed algorithm was applied on the low quality images and compared with the high quality image. The results indicated a reasonable increase in quality and pairwise similarity.

Ajay Boyat and Brijendra Kumar Joshi [13] proposed a novel method for denoising the fingerprint images. The proposed method combines wavelet transform with neighborhood processing for removal of noise. The proposed method is intended to remove Gaussian, Speckle and Salt \&Pepper noise. Experimental analysis was carried out and evaluation of PSNR was done which was improved remarkably.

Ayushi Gupta $\dot{A}$ and Yugshakti Kaushik [2] discussed about various noise removal techniques for de-noising the fingerprint image. The comparative study gives an idea of which noise removal technique is best suitable for removal of Gaussian noise and Salt \& Pepper noise. The experimental analysis is carried out by comparing parameters such as mean, variance and entropy of an image before and after de-noising.
Jie et al [7] proposed a novel approach to deal with False Rejection Rate (FRR). The minutiae feature and orientation field highlight are separated and then intertwined to get a pivot of perpetual quality. Later the entropy pattern is measured by examining the closeness of fragmented fingerprints. Validation is done by comparing the prominent fingerprint image database with the created database which consists of more deficient fingerprint images. The results proved that proposed method is more efficient by reducing FRR.

Consolidated approach about the strategies implemented for improving the recognition by authors- W. Ser X. Jiang [6], K. Hakil et al [14], G. L. Marcialis et al [8], G. L. Marcialis et al [15], G. L. Marcialis et al [20] are discussed below, Size of the memory plays a vital role as we cannot store all collected intra-class variations of user biometric features in real time applications. Also, throughput reduces with the increase in number of templates in the gallery of a user. The common procedures so far adopted for updating the templates are as follows: (1) super template based, in which the input data is always fused to a common single template called "super template" embedding all the information together [16] [17], or (2) instance based, in which the input data is always added as a separate instance to the gallery set of the respective client [23]. Most of the selfupdate online methods have followed super template-based approach [16] [17], However, the offline based self-update [9] and template co-update methods [9] [18] have followed instance-based approach. For updating the templates for intra class variations in constraint environment, FIFO, LFU and clustering algorithms have been modified [19] for implementing template replacement strategy. 


\section{Motivation}

Increase in rejection rate of fingerprint belonging to the genuine user can be addressed by different methods like, Non-Automated, Automated and Adaptive methods.

i. Non-Automated Methods: In this method, whenever there is problem with the matching of user fingerprint, they usually go for multiple (re)enrolment sessions or store multiple templates of the same user in the database. It is time consuming as, many times the same user's fingerprint has to be registered and also it is a manual process.

ii. Automated Methods: In this method, however they adopt subjective and objective process to increase the recognition rate of a fingerprint image such as image enhancement and image restoration. Automated methods use existing mathematical models for improving the image quality to some extent. These methods are not real-time analysis based.

iii. Adaptive methods: In this method, template which is already collected during the time of enrolment phase is updated by a technique of supervised and semisupervised learning methods. In adaptive method, techniques are used to update templates without re-enrolment. It is also called as Adaptive Reference autocorrection system.

In most of the commercial matching algorithm, filters have been used to remove the noises from the fingerprint image at the time of capturing [10]. It has been observed or understood that, the filters have been optimized to remove noises to the maximum extent. Further optimization of filters to remove the noises may degrade the quality of the fingerprint image by removing main features. Hence in this work, adaptive methods are considered for improving the performance against the degradation of fingerprint image due to environmental variation on real-time basis. Adaptive method involves template update method. During the operation, when fingerprint image exhibits intra-class variations, semisupervised learning methods are used to update the user template.

\section{Methodology}

In the proposed Adaptive autocorrection method, initially during enrollment phase, fingerprint images of the user/subject are collected at room temperature and stored as reference fingerprint template in the database. When the user/subject place the finger for authentication, matching score is computed by comparing captured input fingerprint template with the reference fingerprint template stored in the database. If matching score obtained is greater than Threshold value set then, the user/subject is authenticated else rejected.

If the matching score obtained is greater than Threshold value, store the fingerprint template in the cache database. The recognized fingerprint templates are stored in the defined cache in FIFO (first in first out) order for future analysis. This is because, even though the matching score is above Threshold value, due to environmental variations (temperature variations) the minutiae features of the captured input fingerprint image might be deviated from the reference fingerprint image collected during enrollment phase. This deviation leads to varied matching score.

In case of rejection of authentication, the captured input fingerprint image is 
varied beyond certain range due to presence of noise. In such case, corrections need to be applied. While applying correction, first the input rejected fingerprint image of the user/subject must be checked to know whether the rejected input fingerprint image belongs to the same user/subject or to an intruder. In order to find whether the rejected input fingerprint image belongs to the same user/subject, ANN algorithm is used. ANN algorithm classifies the input rejected fingerprint as genuine user/subject or intruder. If the rejected input fingerprint image is of intruder's then, corrections should not be applied on the user's/subject's reference fingerprint template stored in cache database. If the rejected input fingerprint image belongs to the genuine user/subject then, analysis of the fingerprint templates stored in the cache database is performed. Flow chart for the proposed Adaptive Reference auto-correction algorithm is depicted in Figure 1.

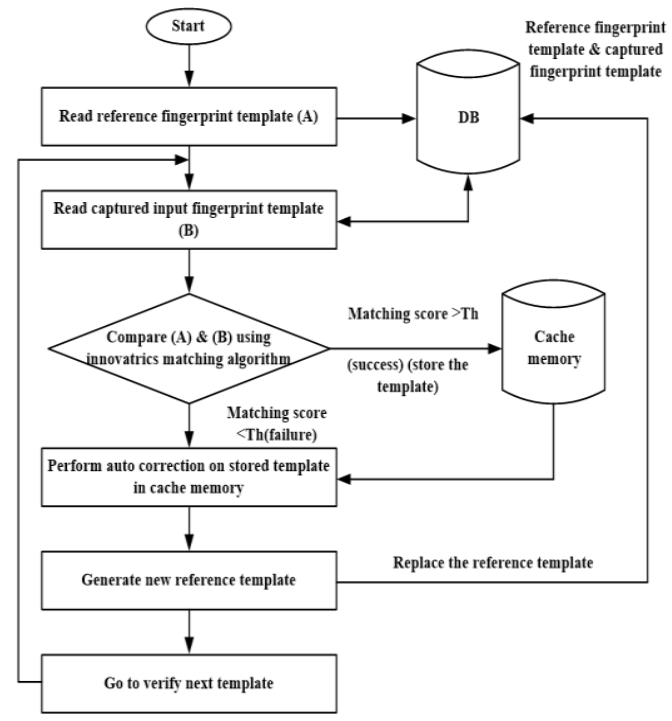

Figure 1: Flow chart of the proposed Adaptive Reference auto-correction algorithm

Fingerprint images of the user are captured using 500DPI optical sensor which is STQC certified to obtain good quality fingerprint image. The captured fingerprint image is used with Innovatrics extractor to extract minutiae template. Then the verification of fingerprint templates is performed using Innovatrics matching algorithm to obtain matching scores. Based on the matching scores, the matching is decided as success or failure. When the user fingerprint is failed to match with the genuine user, the reference template of the genuine user is auto-corrected by Adaptive Reference auto-correction algorithm.

\section{Steps involved in the implementation process are as discussed,}

1. Fingerprint images captured at room temperature are given as input for Innovatrics extractor to obtain ISO 19742 compatible minutiae templates. These templates are stored in flash memory under the respective user ID as file name.

2. The captured input fingerprint images from $25^{\circ} \mathrm{C}$ to $0^{\circ} \mathrm{C}$ of 10 -users are given as input to Innovatrics extractor to obtain equivalent ISO 1974-2 compatible minutiae templates and they are stored in Flash memory as files with user ID and file names affixed with temperature.

Ex: User1 fingerprint templates are stored as, User1.25, User1.24, and User1.23 and so on.

The API call used to extract the template from a bmp file is,

IEngineExportUserTemplate Function Exports user template

IDKIT API intIEngineExportUserTemplate(IENGIN E USER user, IENGINE TEMPLATE FORMAT format, unsigned char * templateData, int * length);

3. The captured input fingerprint template of 10 -users from $25^{\circ} \mathrm{C}$ to $0^{\circ} \mathrm{C}$ are compared using Innovatrics matching algorithm with the respective reference fingerprint template stored in the file to 
obtain matching score. The threshold (Th) used for obtaining the status of matching, success or failure is $\mathrm{Th}=8000$.

4. If matching score is greater than the Threshold value (Th), the result is success (means user Fingerprint is verified). In such case, the ISO template of the captured input Fingerprint image is stored in the cache file. This process is repeated on all ISO template of captured input fingerprint images from $25^{\circ} \mathrm{C}$ to $0^{\circ} \mathrm{C}$.

5. If the matching score is less than the Threshold value (Th), the result is failure (means user Fingerprint is not verified) indicating the ISO template of captured input fingerprint image has failed to match with the reference fingerprint template. At this stage, Adaptive Reference auto-correction algorithm is automatically invoked to correct the reference ISO fingerprint template \& replace.

6. The Adaptive Reference autocorrection algorithm uses the principle of averaging all non-matched or deviated minutiae points of ISO templates stored in the cache file. The ISO templates stored in cache memory are the templates which are succeeded but, with the mix of matched and non-matched minutiae points. Since these templates have succeeded in matching with the reference templates, the number of matching points is more than the number of non-match points satisfying the minimum Threshold of $\mathrm{Th}=8000$.

\section{Results and Discussions}

The proposed Adaptive Reference auto-correction algorithm is implemented on ARM-9 based microcontroller using commercially available Innovatrics extractor and matching algorithm. The processor used is i.MX28 32-bit ARM (ARM926EJ-S CPU) with the speed of $454 \mathrm{MHz}$ Innovatrics algorithm uses ISO
1974-2 minutiae template format to maintain interoperability. In our proposed work we are considering only the genuine user rejection for invoking auto-correction algorithm.

10-users/subjects fingerprint data are considered for validation of the proposed technique. Fingerprints of the users are captured and stored in the database at varied temperature between $25^{\circ} \mathrm{C}$ to $0^{\circ} \mathrm{C}$. First, ISO 1974-2 compatible minutiae templates are extracted from the fingerprint image captured at room temperature and stored in the respective files as reference fingerprint template. Now, expose the user finger for a minimum of 3 minutes in a closed chamber of controlled temperature and capture the fingerprint images by varying temperature from $25^{\circ} \mathrm{C}$ to $0^{\circ} \mathrm{C}$. Total of 26-fingerprint images are captured for every user/subject with a temperature variation of $1^{0} \mathrm{C}$. The verification process is performed for all 10-users/subjects using the captured fingerprint images from $25^{\circ} \mathrm{C}$ to $0^{\circ} \mathrm{C}$ considering one-user at a time. The experiment is conducted using without and with Adaptive Reference auto-correction technique. The results are tabulated in Table 1 and the captured fingerprint images at varied temperature is shown in figure 2.

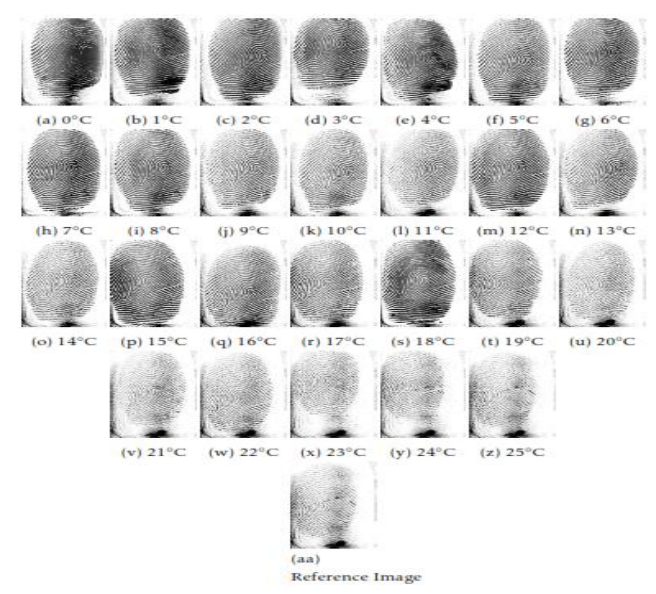

Figure 2: Captured fingerprint images of Subject 1 
Table 1: Subject 1 matching score without and with Auto correction algorithm on microcontroller board

\begin{tabular}{|c|c|c|c|c|c|}
\hline \multicolumn{6}{|c|}{ User1/subject1 } \\
\hline $\begin{array}{l}\text { Te } \\
\mathrm{m} \\
\mathrm{p} . \\
\text { in } \\
{ }^{0} \mathrm{C}\end{array}$ & $\begin{array}{l}\text { Ma } \\
\text { tch } \\
\text { ing } \\
\text { sc } \\
\text { ore }\end{array}$ & $\begin{array}{l}\text { Concl } \\
\text { usion }\end{array}$ & $\begin{array}{l}\mathrm{T} \\
\mathrm{e} \\
\mathrm{m} \\
\mathrm{p} . \\
\text { in } \\
{ }^{0} \mathrm{C}\end{array}$ & $\begin{array}{c}\text { Ma } \\
\text { tch } \\
\text { ing } \\
\text { sc } \\
\text { ore }\end{array}$ & $\begin{array}{l}\text { Con } \\
\text { clusi } \\
\text { on }\end{array}$ \\
\hline 25 & $\begin{array}{c}16 \\
00 \\
0\end{array}$ & $\begin{array}{c}\text { Reco } \\
\text { gnize } \\
\text { d }\end{array}$ & $\begin{array}{l}2 \\
5\end{array}$ & $\begin{array}{c}16 \\
00 \\
0\end{array}$ & $\begin{array}{l}\text { Rec } \\
\text { ogni } \\
\text { zed }\end{array}$ \\
\hline 24 & $\begin{array}{c}14 \\
00 \\
0\end{array}$ & $\begin{array}{c}\text { Reco } \\
\text { gnize } \\
\text { d }\end{array}$ & $\begin{array}{l}2 \\
4\end{array}$ & $\begin{array}{c}14 \\
00 \\
0\end{array}$ & $\begin{array}{l}\text { Rec } \\
\text { ogni } \\
\text { zed }\end{array}$ \\
\hline 23 & $\begin{array}{c}15 \\
00 \\
0 \\
\end{array}$ & $\begin{array}{c}\text { Reco } \\
\text { gnize } \\
\text { d }\end{array}$ & $\begin{array}{l}2 \\
3\end{array}$ & $\begin{array}{c}15 \\
00 \\
0 \\
\end{array}$ & $\begin{array}{l}\text { Rec } \\
\text { ogni } \\
\text { zed }\end{array}$ \\
\hline 22 & $\begin{array}{c}12 \\
00 \\
0\end{array}$ & $\begin{array}{c}\text { Reco } \\
\text { gnize } \\
\text { d }\end{array}$ & $\begin{array}{l}2 \\
2\end{array}$ & $\begin{array}{c}12 \\
00 \\
0\end{array}$ & $\begin{array}{l}\text { Rec } \\
\text { ogni } \\
\text { zed }\end{array}$ \\
\hline 21 & $\begin{array}{c}13 \\
56 \\
7 \\
\end{array}$ & $\begin{array}{c}\text { Reco } \\
\text { gnize } \\
d \\
\end{array}$ & $\begin{array}{l}2 \\
1\end{array}$ & $\begin{array}{c}13 \\
56 \\
7 \\
\end{array}$ & $\begin{array}{l}\text { Rec } \\
\text { ogni } \\
\text { zed }\end{array}$ \\
\hline 20 & $\begin{array}{c}14 \\
55 \\
7\end{array}$ & $\begin{array}{c}\text { Reco } \\
\text { gnize } \\
\text { d }\end{array}$ & $\begin{array}{l}2 \\
0\end{array}$ & $\begin{array}{c}14 \\
55 \\
7\end{array}$ & $\begin{array}{l}\operatorname{Rec} \\
\text { ogni } \\
\text { zed }\end{array}$ \\
\hline 19 & $\begin{array}{c}15 \\
03 \\
4 \\
\end{array}$ & $\begin{array}{c}\text { Reco } \\
\text { gnize } \\
d \\
\end{array}$ & $\begin{array}{l}1 \\
9\end{array}$ & $\begin{array}{c}15 \\
03 \\
4\end{array}$ & $\begin{array}{l}\text { Rec } \\
\text { ogni } \\
\text { zed }\end{array}$ \\
\hline 18 & $\begin{array}{c}12 \\
96 \\
0\end{array}$ & $\begin{array}{c}\text { Reco } \\
\text { gnize } \\
d\end{array}$ & $\begin{array}{l}1 \\
8\end{array}$ & $\begin{array}{c}12 \\
96 \\
0\end{array}$ & $\begin{array}{l}\text { Rec } \\
\text { ogni } \\
\text { zed }\end{array}$ \\
\hline 17 & $\begin{array}{c}13 \\
25 \\
0 \\
\end{array}$ & $\begin{array}{c}\text { Reco } \\
\text { gnize } \\
d \\
d\end{array}$ & $\begin{array}{l}1 \\
7\end{array}$ & $\begin{array}{c}13 \\
25 \\
0 \\
\end{array}$ & $\begin{array}{l}\text { Rec } \\
\text { ogni } \\
\text { zed }\end{array}$ \\
\hline 16 & $\begin{array}{c}13 \\
75 \\
7 \\
\end{array}$ & $\begin{array}{c}\text { Reco } \\
\text { gnize } \\
d\end{array}$ & $\begin{array}{l}1 \\
6\end{array}$ & $\begin{array}{c}13 \\
75 \\
7 \\
\end{array}$ & $\begin{array}{l}\text { Rec } \\
\text { ogni } \\
\text { zed }\end{array}$ \\
\hline 15 & $\begin{array}{c}12 \\
86 \\
5\end{array}$ & $\begin{array}{c}\text { Reco } \\
\text { gnize } \\
\text { d }\end{array}$ & $\begin{array}{l}1 \\
5\end{array}$ & $\begin{array}{c}12 \\
86 \\
5\end{array}$ & $\begin{array}{l}\text { Rec } \\
\text { ogni } \\
\text { zed }\end{array}$ \\
\hline 14 & $\begin{array}{c}13 \\
36 \\
5\end{array}$ & $\begin{array}{c}\text { Reco } \\
\text { gnize } \\
\text { d }\end{array}$ & $\begin{array}{l}1 \\
4\end{array}$ & $\begin{array}{c}13 \\
36 \\
5\end{array}$ & $\begin{array}{l}\text { Rec } \\
\text { ogni } \\
\text { zed }\end{array}$ \\
\hline
\end{tabular}

\begin{tabular}{|c|c|c|c|c|c|}
\hline 13 & $\begin{array}{c}12 \\
62 \\
3\end{array}$ & $\begin{array}{c}\text { Reco } \\
\text { gnize } \\
\text { d }\end{array}$ & $\begin{array}{l}1 \\
3\end{array}$ & $\begin{array}{c}12 \\
62 \\
3\end{array}$ & $\begin{array}{l}\text { Rec } \\
\text { ogni } \\
\text { zed }\end{array}$ \\
\hline 12 & $\begin{array}{c}12 \\
12 \\
2\end{array}$ & $\begin{array}{c}\text { Reco } \\
\text { gnize } \\
\text { d }\end{array}$ & $\begin{array}{l}1 \\
2\end{array}$ & $\begin{array}{c}12 \\
12 \\
2\end{array}$ & $\begin{array}{l}\text { Rec } \\
\text { ogni } \\
\text { zed }\end{array}$ \\
\hline 11 & $\begin{array}{c}12 \\
42 \\
7\end{array}$ & $\begin{array}{c}\text { Reco } \\
\text { gnize } \\
\text { d }\end{array}$ & $\begin{array}{l}1 \\
1\end{array}$ & $\begin{array}{c}12 \\
42 \\
7\end{array}$ & $\begin{array}{l}\text { Rec } \\
\text { ogni } \\
\text { zed }\end{array}$ \\
\hline 10 & $\begin{array}{c}11 \\
62 \\
2\end{array}$ & $\begin{array}{c}\text { Reco } \\
\text { gnize } \\
\text { d }\end{array}$ & $\begin{array}{l}1 \\
0\end{array}$ & $\begin{array}{c}11 \\
62 \\
2\end{array}$ & $\begin{array}{l}\operatorname{Rec} \\
\text { ogni } \\
\text { zed }\end{array}$ \\
\hline 9 & $\begin{array}{c}11 \\
35 \\
6\end{array}$ & $\begin{array}{c}\text { Reco } \\
\text { gnize } \\
\text { d }\end{array}$ & 9 & $\begin{array}{c}11 \\
35 \\
6\end{array}$ & $\begin{array}{l}\text { Rec } \\
\text { ogni } \\
\text { zed }\end{array}$ \\
\hline 8 & $\begin{array}{c}11 \\
53 \\
4\end{array}$ & $\begin{array}{c}\text { Reco } \\
\text { gnize } \\
\text { d }\end{array}$ & 8 & $\begin{array}{c}11 \\
53 \\
4\end{array}$ & $\begin{array}{l}\text { Rec } \\
\text { ogni } \\
\text { zed }\end{array}$ \\
\hline 7 & $\begin{array}{c}10 \\
89 \\
7\end{array}$ & $\begin{array}{c}\text { Reco } \\
\text { gnize } \\
\text { d }\end{array}$ & 7 & $\begin{array}{c}10 \\
89 \\
7\end{array}$ & $\begin{array}{l}\text { Rec } \\
\text { ogni } \\
\text { zed }\end{array}$ \\
\hline 6 & $\begin{array}{c}10 \\
21 \\
1\end{array}$ & $\begin{array}{c}\text { Reco } \\
\text { gnize } \\
\text { d }\end{array}$ & 6 & $\begin{array}{c}10 \\
21 \\
1\end{array}$ & $\begin{array}{l}\operatorname{Rec} \\
\text { ogni } \\
\text { zed }\end{array}$ \\
\hline 5 & $\begin{array}{c}10 \\
34 \\
5\end{array}$ & $\begin{array}{c}\text { Reco } \\
\text { gnize } \\
\text { d }\end{array}$ & 5 & $\begin{array}{c}10 \\
34 \\
5\end{array}$ & $\begin{array}{l}\text { Rec } \\
\text { ogni } \\
\text { zed }\end{array}$ \\
\hline 4 & $\begin{array}{l}96 \\
78\end{array}$ & $\begin{array}{c}\text { Reco } \\
\text { gnize } \\
\text { d }\end{array}$ & 4 & $\begin{array}{l}96 \\
78\end{array}$ & $\begin{array}{l}\text { Rec } \\
\text { ogni } \\
\text { zed }\end{array}$ \\
\hline 3 & $\begin{array}{l}87 \\
98\end{array}$ & $\begin{array}{c}\text { Reco } \\
\text { gnize } \\
\text { d }\end{array}$ & 3 & $\begin{array}{l}87 \\
98\end{array}$ & $\begin{array}{l}\operatorname{Rec} \\
\text { ogni } \\
\text { zed }\end{array}$ \\
\hline 2 & $\begin{array}{l}81 \\
02\end{array}$ & $\begin{array}{c}\text { Reco } \\
\text { gnize } \\
\text { d }\end{array}$ & 2 & $\begin{array}{l}81 \\
02\end{array}$ & $\begin{array}{l}\text { Rec } \\
\text { ogni } \\
\text { zed }\end{array}$ \\
\hline 1 & 0 & $\begin{array}{c}\text { Not } \\
\text { Reco } \\
\text { gnize } \\
\text { d }\end{array}$ & 1 & $\begin{array}{c}14 \\
00 \\
0\end{array}$ & $\begin{array}{l}\text { Rec } \\
\text { ogni } \\
\text { zed }\end{array}$ \\
\hline 0 & 0 & $\begin{array}{c}\text { Not } \\
\text { Reco } \\
\text { gnize } \\
\text { d }\end{array}$ & 0 & $\begin{array}{c}13 \\
12 \\
0\end{array}$ & $\begin{array}{l}\text { Rec } \\
\text { ogni } \\
\text { zed }\end{array}$ \\
\hline
\end{tabular}

(a) without auto-correction technique (b) with auto-correction technique 
From Table1 (a), the matching score obtained for user1/subject1 shows that, the fingerprint image has satisfied to be recognized until the temperature of $1{ }^{\circ} \mathrm{C}$. This fingerprint image is quite stable against the variation of temperature. Even though the matching scores are decreased due to the decrease in temperature, it satisfies the minimum matching score requirement of 8000 , hence they are declared as recognized. But below $1{ }^{\circ} \mathrm{C}$ the matching score is less than 8000 , hence they are declared as not recognized.

Success rate: $92.3 \%$; Failure rate: $7.7 \%$

From Table1(b), the matching scores obtained after applying Adaptive Reference auto-correction technique shows that, even for temperature below $1^{0} \mathrm{C}$ the matching scores have improved above 8000 hence, declared recognized. At $1^{0} \mathrm{C}$, the matching points have been increased to 14000 , due to the correction applied for the reference fingerprint template.

Success rate: $100 \%$; Failure rate: $0 \%$

Similarly, analysis is carried out for rest of the users. As all the values cannot be shown in detail due to spacing, a consolidated matching scores of all the 10 -suers/subject is tabulated in the Table 2 and Table 3.

Table 2: Matching scores of 10users/subjects without Adaptive auto-correction

\begin{tabular}{|c|c|c|c|c|c|c|c|c|c|c|c|c|c|c|c|c|c|c|c|c|c|}
\hline $\mathrm{T}$ & Mat & hing & cores & & & & & & & & 7 & $\begin{array}{r}89 \\
7\end{array}$ & 0 & $\begin{array}{l}10 \\
21\end{array}$ & $\begin{array}{l}85 \\
12\end{array}$ & $\begin{array}{r}97 \\
2\end{array}$ & $\begin{array}{r}42 \\
1\end{array}$ & 0 & $\begin{array}{r}24 \\
1\end{array}$ & $\begin{array}{r}39 \\
2\end{array}$ & $\begin{array}{r}810 \\
5\end{array}$ \\
\hline $\begin{array}{l}e \\
m \\
p\end{array}$ & & & & & & & & & & & 6 & $\begin{array}{r}10 \\
21 \\
1\end{array}$ & 0 & $\begin{array}{l}98 \\
34\end{array}$ & 0 & $\begin{array}{r}12 \\
67 \\
1\end{array}$ & $\begin{array}{r}11 \\
10 \\
3\end{array}$ & 0 & $\begin{array}{r}11 \\
72 \\
3\end{array}$ & $\begin{array}{r}10 \\
93 \\
0\end{array}$ & 0 \\
\hline $\begin{array}{l}\mathrm{i} \\
\mathrm{n}\end{array}$ & & & & & & & & & & & 5 & $\begin{array}{r}10 \\
34 \\
5\end{array}$ & 0 & $\begin{array}{l}95 \\
78\end{array}$ & 0 & $\begin{array}{r}12 \\
01 \\
0\end{array}$ & $\begin{array}{r}10 \\
51 \\
9\end{array}$ & 0 & $\begin{array}{r}11 \\
61 \\
0\end{array}$ & $\begin{array}{l}92 \\
92\end{array}$ & 0 \\
\hline $\begin{array}{l}0 \\
\mathrm{C}\end{array}$ & $\begin{array}{l}\text { Su } \\
\text { b1 }\end{array}$ & $\begin{array}{l}\mathrm{Su} \\
\text { b2 }\end{array}$ & $\begin{array}{l}\text { Su } \\
\text { b3 }\end{array}$ & $\begin{array}{l}\mathrm{Su} \\
\text { b4 }\end{array}$ & $\begin{array}{l}\mathrm{Su} \\
\text { b5 }\end{array}$ & $\begin{array}{l}\mathrm{Su} \\
\text { b6 }\end{array}$ & $\begin{array}{l}\text { Su } \\
\text { b7 }\end{array}$ & $\begin{array}{l}\mathrm{Su} \\
\text { b8 }\end{array}$ & $\begin{array}{l}\text { Su } \\
\text { b9 }\end{array}$ & $\begin{array}{l}\text { Sul } \\
10\end{array}$ & & $\begin{array}{l}96 \\
78\end{array}$ & 0 & $\begin{array}{l}89 \\
67\end{array}$ & 0 & $\begin{array}{r}11 \\
79 \\
2\end{array}$ & $\begin{array}{r}11 \\
61 \\
0\end{array}$ & 0 & $\begin{array}{r}11 \\
89 \\
2\end{array}$ & $\begin{array}{l}87 \\
01\end{array}$ & 0 \\
\hline $\begin{array}{l}2 \\
5\end{array}$ & $\begin{array}{r}16 \\
00 \\
0\end{array}$ & $\begin{array}{r}14 \\
23 \\
8\end{array}$ & $\begin{array}{r}12 \\
56 \\
9 \\
\end{array}$ & $\begin{array}{r}12 \\
98 \\
8 \\
\end{array}$ & $\begin{array}{r}13 \\
25 \\
2 \\
\end{array}$ & $\begin{array}{r}14 \\
59 \\
1 \\
\end{array}$ & $\begin{array}{r}12 \\
01 \\
0 \\
\end{array}$ & $\begin{array}{r}15 \\
20 \\
1 \\
\end{array}$ & $\begin{array}{r}13 \\
21 \\
0 \\
\end{array}$ & $\begin{array}{r}12 \\
9 \\
\end{array}$ & & $\begin{array}{l}87 \\
98\end{array}$ & 0 & $\begin{array}{l}83 \\
56\end{array}$ & 0 & $\begin{array}{r}11 \\
42 \\
1\end{array}$ & $\begin{array}{r}11 \\
10 \\
9\end{array}$ & 0 & $\begin{array}{r}10 \\
56 \\
2\end{array}$ & 0 & 0 \\
\hline
\end{tabular}




\begin{tabular}{|r|r|r|r|r|r|r|r|r|r|r|} 
& 81 & & 81 & & 01 & 10 & & 10 & & \\
2 & 02 & 0 & 20 & 0 & 3 & 2 & 0 & 9 & 0 & 0 \\
\hline & & & & & 92 & 97 & & 10 & & \\
& & & & & 01 & 12 & & 62 & & \\
\hline & 0 & 0 & 0 & 0 & 8 & 0 & 0 & 0 & 0 & 0 \\
\hline 0 & 0 & 0 & 0 & 0 & 93 & 92 & & 97 & & \\
& & & & & & \\
\end{tabular}

score on success but, zero matching score on failure.

Figure 2 and Figure 3 shows the plot for matching scores tabulated in Table $2 \&$ Table 3.

Table 3: Matching scores of 10users/subjects with Adaptive autocorrection

\begin{tabular}{|c|c|c|c|c|c|c|c|c|c|}
\hline \multirow{2}{*}{$\begin{array}{l}\text { Temp. } \\
\text { in }{ }^{0} \mathrm{C}\end{array}$} & \multicolumn{9}{|c|}{ Matching scores } \\
\hline & Sub1 & Sub2 & Sub3 & Sub4 & Sub5 & Sub6 & Sub7 & Sub8 & Sub \\
\hline 25 & 16000 & 14238 & 12569 & 12988 & 13252 & 14591 & 12010 & 15201 & 132 \\
\hline 24 & 14000 & 14650 & 12345 & 12712 & 14234 & 13891 & 11672 & 14751 & 129 \\
\hline 23 & 15000 & 13678 & 12785 & 13105 & 14521 & 14230 & 11102 & 14201 & $126 !$ \\
\hline 22 & 12000 & 13567 & 12432 & 12867 & 13987 & 14981 & 11754 & 15104 & 131( \\
\hline 21 & 13567 & 13722 & 11987 & 12610 & 13671 & 14421 & 10109 & 14892 & 1286 \\
\hline 20 & 14557 & 13210 & 12112 & 12823 & 14012 & 13891 & 9201 & 15452 & $124 !$ \\
\hline 19 & 15034 & 12567 & 11723 & 12590 & 13891 & 14190 & 8348 & 14691 & 111( \\
\hline 18 & 12960 & 12311 & 11465 & 12301 & 13201 & 13691 & 8521 & 14301 & $129 !$ \\
\hline 17 & 13250 & 11785 & 12023 & 12452 & 13634 & 13201 & 8104 & 13792 & 1238 \\
\hline 16 & 13757 & 11201 & 11678 & 11569 & 13802 & 12607 & 11892 & 14288 & 1269 \\
\hline 15 & 12865 & 10862 & 11124 & 12341 & 13103 & 12981 & 11341 & 13432 & 1193 \\
\hline 14 & 13365 & 10621 & 10987 & 12478 & 12956 & 12510 & 11572 & 12845 & 1173 \\
\hline 13 & 12623 & 9942 & 10673 & 11201 & 13450 & 13612 & 10902 & 13102 & 1204 \\
\hline 12 & 12122 & 10102 & 10110 & 11834 & 12780 & 12782 & 10793 & 12642 & 1120 \\
\hline 11 & 12427 & 9231 & 10451 & 11390 & 12431 & 12102 & 10201 & 12904 & 117( \\
\hline 10 & 11622 & 8329 & 10213 & 10856 & 12941 & 11823 & 10411 & 12329 & $108^{\circ}$ \\
\hline 9 & 11356 & 13500 & 9981 & 10201 & 13450 & 12002 & 9612 & 12744 & 114 \\
\hline 8 & 11534 & 12753 & 9946 & 9451 & 12301 & 11750 & 9810 & 11893 & $107^{\prime}$ \\
\hline 7 & 10897 & 13021 & 1021 & 8512 & 11972 & 11421 & 8651 & 12241 & 103 \\
\hline 6 & 10211 & 12236 & 9834 & 11023 & 12671 & 11103 & 8211 & 11723 & $109:$ \\
\hline 5 & 10345 & 11301 & 9578 & 11452 & 12010 & 10519 & 0 & 11610 & 929 \\
\hline 4 & 9678 & 9086 & 8967 & 10367 & 11792 & 11610 & 0 & 11892 & 870 \\
\hline 3 & 8798 & 8992 & 8356 & 9782 & 11421 & 11109 & 0 & 10562 & 1256 \\
\hline 2 & 8102 & 8102 & 8120 & 8210 & 10013 & 10102 & 0 & 10329 & $114 !$ \\
\hline 1 & 14000 & 0 & 11245 & 0 & 92018 & 97120 & 0 & 10620 & 118 \\
\hline 0 & 13120 & 0 & 10564 & 0 & 9329 & 9210 & 0 & 9782 & $110^{\circ}$ \\
\hline
\end{tabular}

From the Tables 1,2 and 3 it can be observed that, whenever the matching is success matching scores are recorded but, whenever the matching is failed there are no matching scores recorded. This is because, the commercial Innovatrics algorithm provides matching

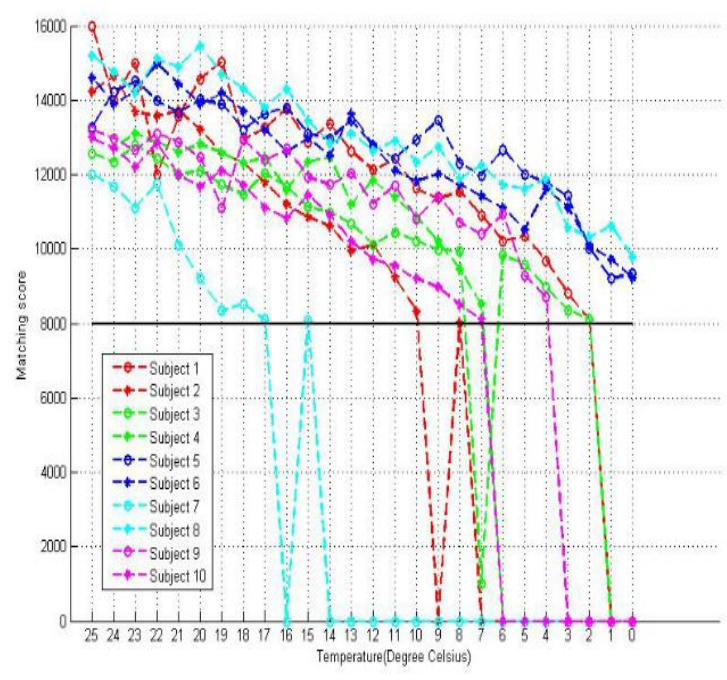

fंg्sure 2: Graph of matching cores versus temperature of 10sers 1 subjects without Adaptive atyto correction technique.

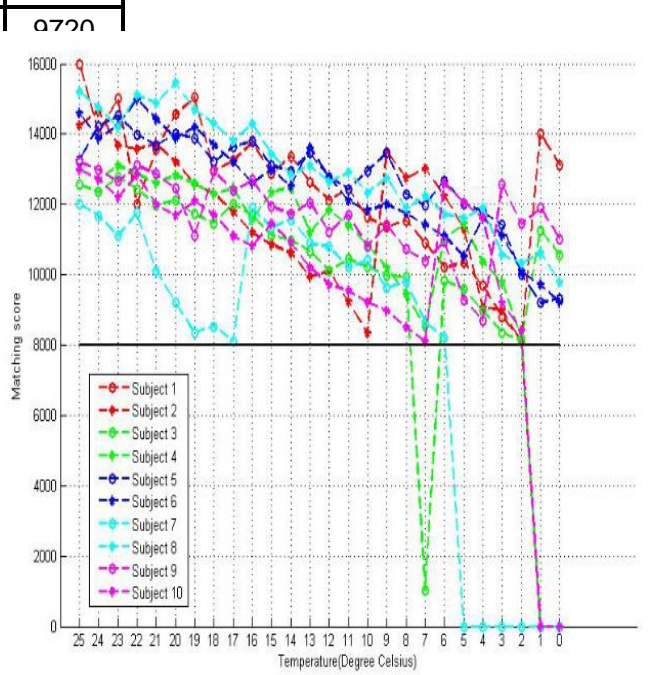

Figure 3: Graph of matching scores versus temperature of 10users/subjects with Adaptive autocorrection technique.

The results obtained for all 10-users without Adaptive auto-correction and 
with auto-correction technique indicate that, there is a significant improvement in the matching score and success rate after applying Adaptive auto-correction technique. Summary of the results are tabulated in Table 4.

Table 4: Summary of success and failure rate

\begin{tabular}{|c|c|c|c|c|c|c|c|c|}
\hline $\begin{array}{c}\text { Tot } \\
\text { al } \\
\text { No. } \\
\text { of } \\
\text { use }\end{array}$ & \multicolumn{4}{|c|}{$\begin{array}{l}\text { Without Adaptive } \\
\text { Reference auto- } \\
\text { correction }\end{array}$} & \multicolumn{4}{|c|}{$\begin{array}{l}\text { With Adaptive } \\
\text { Reference auto- } \\
\text { correction }\end{array}$} \\
\hline \multirow{7}{*}{10} & $\mathrm{~N}$ & In & $\mathrm{N}$ & In & $\mathrm{N}$ & $\ln$ & $\mathrm{N}$ & \\
\hline & 0. & pe & 0. & pe & O. & pe & 0. & pe \\
\hline & Of & rc & of & rc & $\begin{array}{l}\text { Of } \\
\text { su }\end{array}$ & rc & of & rc \\
\hline & cC & en & fai & en & cC & en & fai & en \\
\hline & es & ta & lur & ta & es & ta & lur & ta \\
\hline & $\mathrm{s}$ & ge & e & ge & $\mathrm{s}$ & ge & e & ge \\
\hline & 3 & $\begin{array}{l}30 \\
\%\end{array}$ & 7 & $\begin{array}{l}70 \\
\%\end{array}$ & 6 & $\begin{array}{l}60 \\
\%\end{array}$ & 4 & $\begin{array}{l}40 \\
\%\end{array}$ \\
\hline
\end{tabular}

Table 4 indicates the performance of 10-users on an average. Without Adaptive Reference auto-correction technique, 3-users or $30 \%$ of user fingerprint images are matched or success for temperature range $25^{\circ} \mathrm{C}$ to $0^{0} \mathrm{C}$. But, 7-users or $70 \%$ of user fingerprint images are failed to recognize at different temperature levels as the temperature varies. Hence, there is $30 \%$ success and $70 \%$ failure on an average for 10-users put together when algorithm is executed on microcontroller board with ISO 1974-2 Innovatrics extractor and matcher.

With Adaptive Reference autocorrection technique, result shows 6users or $60 \%$ of user fingerprints matched, but only 4 -users or $40 \%$ of user fingerprints as failed to match for temperature range from $25^{\circ} \mathrm{C}$ to $0^{\circ} \mathrm{C}$. There is $60 \%$ success and $40 \%$ failure on an average for all 10-users after applying Adaptive Reference auto-correction technique with Innovatrics commercial algorithm and ISO 1974-2 templates. Thus, there is an improvement of $30 \%$ in the success rate for the temperature range $25^{\circ} \mathrm{C}$ to $0^{\circ} \mathrm{C}$ on an average.

Analysis is carried out for the temperature variation based on the seasons and regions. Based on the regions, temperature variations may be few degrees like $20^{\circ} \mathrm{C}, 18^{\circ} \mathrm{C}, 15^{\circ} \mathrm{C}, 12^{\circ} \mathrm{C}$, $10^{\circ} \mathrm{C}, 8^{0} \mathrm{C}, 6^{\circ} \mathrm{C}$ etc. Considering the regional variations in temperature, the results are analyzed for various temperature ranges to show the performance. The results are analyzed for various temperature ranges like $25^{\circ} \mathrm{C}$ to $20^{\circ} \mathrm{C}, 25^{\circ} \mathrm{C}$ to $15^{\circ} \mathrm{C}, 25^{\circ} \mathrm{C}$ to $10^{\circ} \mathrm{C}$ etc. as show in the Table 5 . 
Table 5: Success rates and Failure rates analysis without and with Adaptive auto-correction technique. correction and with Adaptive Reference auto-correction are shown in Figure 4.

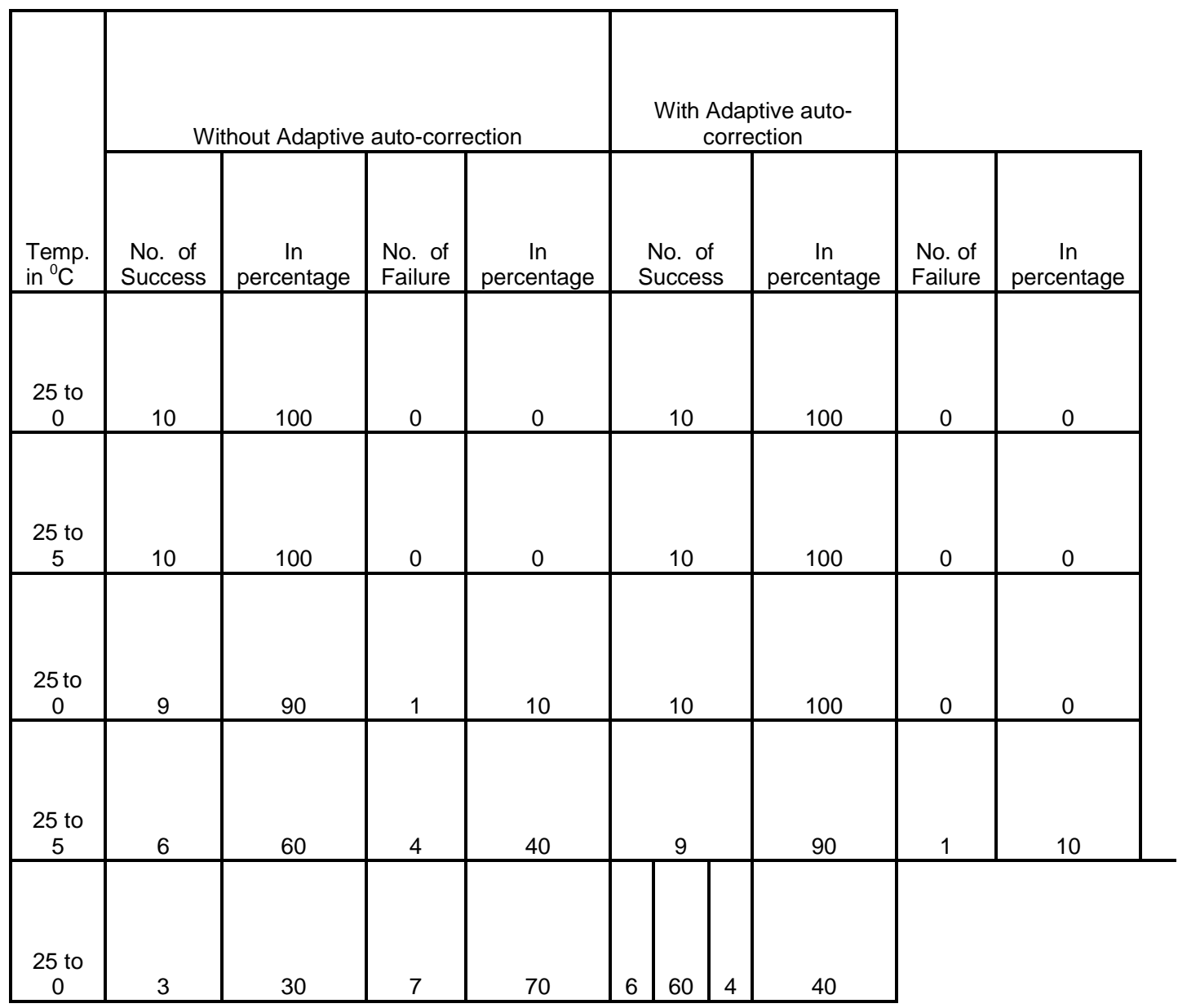

From Table 5 it can be noticed that, there is a significant improvement in the success rate and $100 \%$ matching up to $10^{\circ} \mathrm{C}$ and almost $90 \%$ success up to $5^{\circ} \mathrm{C}$, after applying Adaptive Reference autocorrection technique. So, in the regions where the temperature variation from $25^{\circ} \mathrm{C}$ to $5^{0} \mathrm{C}$ affected the recognition rate, there is significant improvement in recognition rate.

The graph of Success rates and Failure rates, without Adaptive Reference auto- 


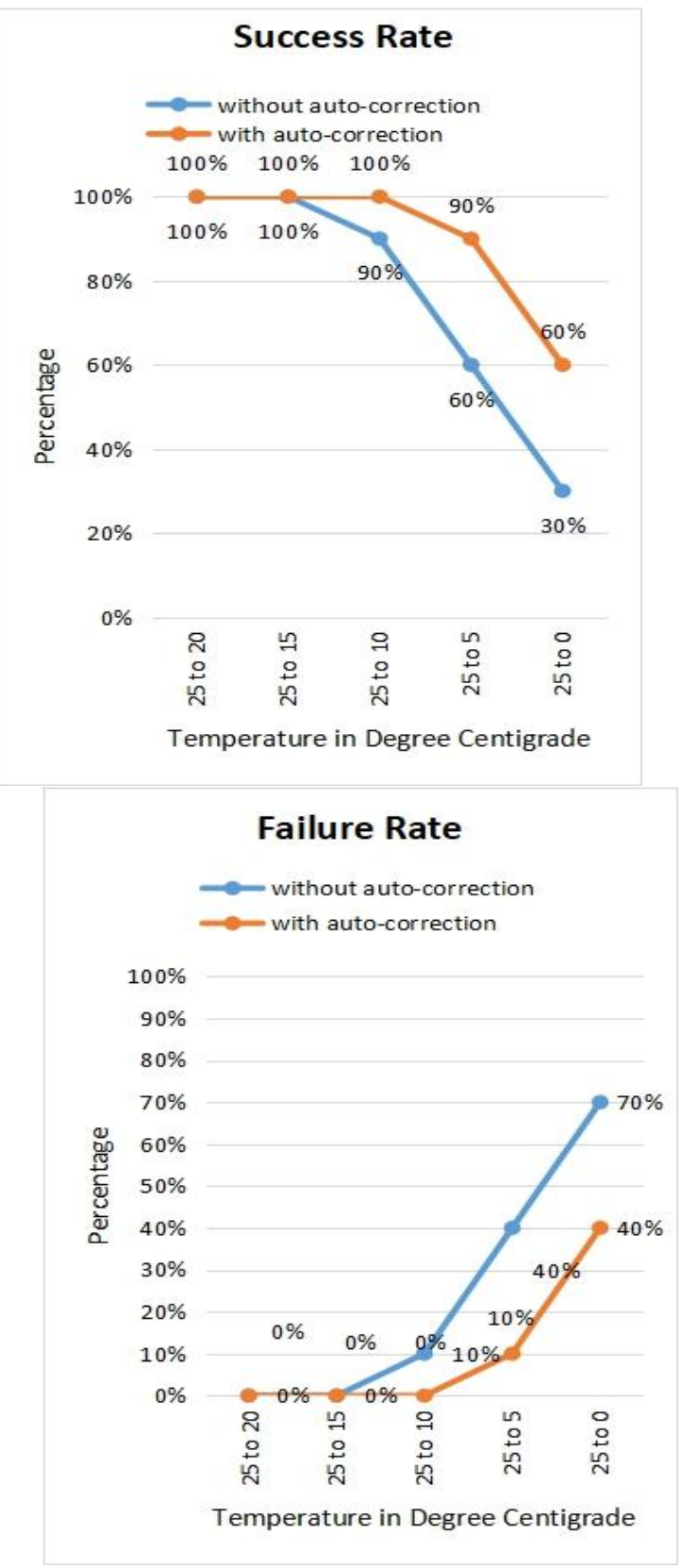

Figure 4: Graph of Success rates and Failure rates with and without Adaptive auto-correction technique between $25^{\circ} \mathrm{C}$ to $5^{\circ} \mathrm{C}$.

\section{Conclusion}

The proposed Adaptive autocorrection technique which mainly focused on biometric time-attendance system was implemented on commercially available biometric device to verify the credibility of the technique. The results and performance analysis for the temperature variation based on the seasons and regions were carried out. It is noticed that, there is a significant improvement in the success rate and almost $100 \%$ matching for temperature variation from $25^{\circ} \mathrm{C}$ to $10^{\circ} \mathrm{C}$ and $90 \%$ matching for temperature variation from $25^{\circ} \mathrm{C}$ to $5^{\circ} \mathrm{C}$. Even, in the case when temperature falls below $5^{0} \mathrm{C}$, there is a significant improvement in the success rate up to $60 \%$.

From the graphs it is observed that, the proposed adaptive auto-correction technique is quite consistent and achieves an improvement in the recognition rate of biometric systems against the variation of temperature.

\section{References}

\subsection{Journal Article}

[1] Saini, Kalpana, and M. Dewal. "Designing of a Virtual System with Fingerprint Security by considering many Security threats." International Journal of Computer Applications IJCA 3 (2010): 25-31.

[2] Ayushi Gupta, Yugshakti Kaushik “A Comparative Study of Noise Removal Techniques", International Journal of Current Engineering and Technology, Vol.4, No.6, 2014

[3] P. Thejaswini, R. Srikantaswamy and A.S. Manjunatha, "Environmental Impact on Biometric Traits and Methods to Improve Biometric Recognition System”, 2015, 10.3850/978-981-09-6200-5_32.

[4] Theofanos, Mary, "Does habituation affect fingerprint quality?" CHI'06 Extended Abstracts on Human Factors in Computing Systems. ACM, 2006.

[5] Speir, Jacqueline, Jack Hietpas. "Frequency Filtering To Suppress Background Noise In Fingerprint Evidence: Quantifying The Fidelity Of Digitally Enhanced Fingerprint Images", Forensic Science International 242, Pp: 94-102, 2014

[6] W. Ser X. Jiang. Online fingerprint template improvement. In In IEEE Trans. PAMI, volume 24, pages 1121-1126, 2002. [cited at p. $39,41,42,44,50,53,58]$ 
[7] Jie Zhang, Xiao-jun Jing, Na Chen, Jian-li Wang, "Incomplete fingerprint recognition based on feature fusion and pattern entropy", the Journal of China Universities of Posts and Telecommunications, Vol. 20, No.3, pp. 121128,2013

[8] G. L. Marcialis F. Roli. Semi-supervised pcabased face recognition using self-training. In Joint IAPR Int. Work. on S+SSPR06, volume 4109 , pages $560-568,2006$. [cited at p. 35, $36,39,44,50,53,99]$

[9] Fabio Roli and Gian Luca Marcialis. Semisupervised pca-based face recognition using self-training. In Joint IAPR International Workshops on Statistical Techniques in Pattern Recognition (SPR) and Structural and Syntactic Pattern Recognition (SSPR), pages 560-568. Springer, 2006.

[10] Thejaswini P, Srikantaswamy R, Manjunatha A. "Novel Adaptive Auto-Correction Technique for Enhanced Fingerprint Recognition", International Journal of Advanced Computer Science and Applications, 2019.

\subsection{Book}

[11] D. Maltoni, Dmaio, A.K. Jain, S. Prabhakar, "Hand book of Fingerprint recognition", springer, 2003.

\subsection{Conference Proceedings}

[12] Thejaswini P, Srikantaswmay R. and Manjunatha A S, "Impact of Fingerprint image quality on matching score", Lect. Notes in Networks, Syst., Vol. 14, D. S. Guru (Eds): Proceedings of International Conference on Cognition and Recognition, Springer Nature,2018, DOI: 10.1007/978981-10-5146-3, Series ISSN: 2367-3370

[13] Ajay Boyat , Brijendra Kumar "Image Denoising using Wavelet Transform and Median Filtering", in proceeding conference on IEEE , 2013

[14] K. Hakil A. Jain C. Ryu. Template adaptation-based fingerprint verification. In Proc. Of International Conference on Pattern Recognition (ICPR), volume 4, pages 582585, 2006. [cited at p. 37, 39, 41, 42, 44, 50, $53,56,57,58,63]$

[15] G.L. Marcialis F. Roli, L. Didaci. Template co-update in multimodal biometric systems. In IEEE/IAPR 2nd International Conference on Biometrics (ICB), volume 4642, pages 1194-1202, 2007. [cited at p. 43, 44, 50, 75, $76,78,81]$

[16] Xudong Jiang and Wee Ser. Online fingerprint template improvement. IEEE
Transactions on Pattern Analysis and Machine Intelligence, 24(8):1121-1126, 2002

[17] Choonwoo Ryu, Hakil Kim, and Anil K Jain. Template adaptation-based fingerprint verification. In Pattern Recognition, 2006. ICPR 2006. 18th International Conference on, volume 4, pages 582-585. IEEE, 2006.

[18] Fabio Roli, Luca Didaci, and Gian Luca Marcialis. Template co-update in multimodal biometric systems. In International Conference on Biometrics, pages 1194-1202. Springer, 2007.

[19] Biagio Freni, Gian Luca Marcialis, and Fabio Roli. Replacement algorithms for fingerprint template update. In International Conference Image Analysis and Recognition, pages 884 893. Springer, 2008.

[20] G.L. Marcialis B. Freni and F. Roli. Replacement algorithms for fingerprint template update. In ICIAR08, pages 884-893. Springer LNCS5112, 2008. [cited at p. 44]

[21] www.supremainc.com

[22] www.morpho.com

[23] www.innovatrics.com

[24] www.neurotechnology.com/megamatcher.ht $\mathrm{ml}$

[25] www.secugen.com

\section{Authors}

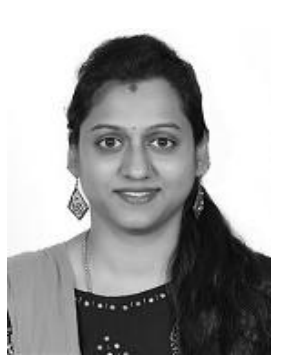

Dr Thejaswini $P$ completed BE (ECE) in 2006, M.Tech (VLSI design \& Embedded systems) in 2008 and $\mathrm{PhD}$ (Embedded Systems) in 2018 from Visvesvaraya Technological University, Belgaum. Presently working as Assistant Professor in the department of Electronics \& Communication at JSS Academy of Technical Education, Bangalore. She has published research papers in international/national journals and conference 


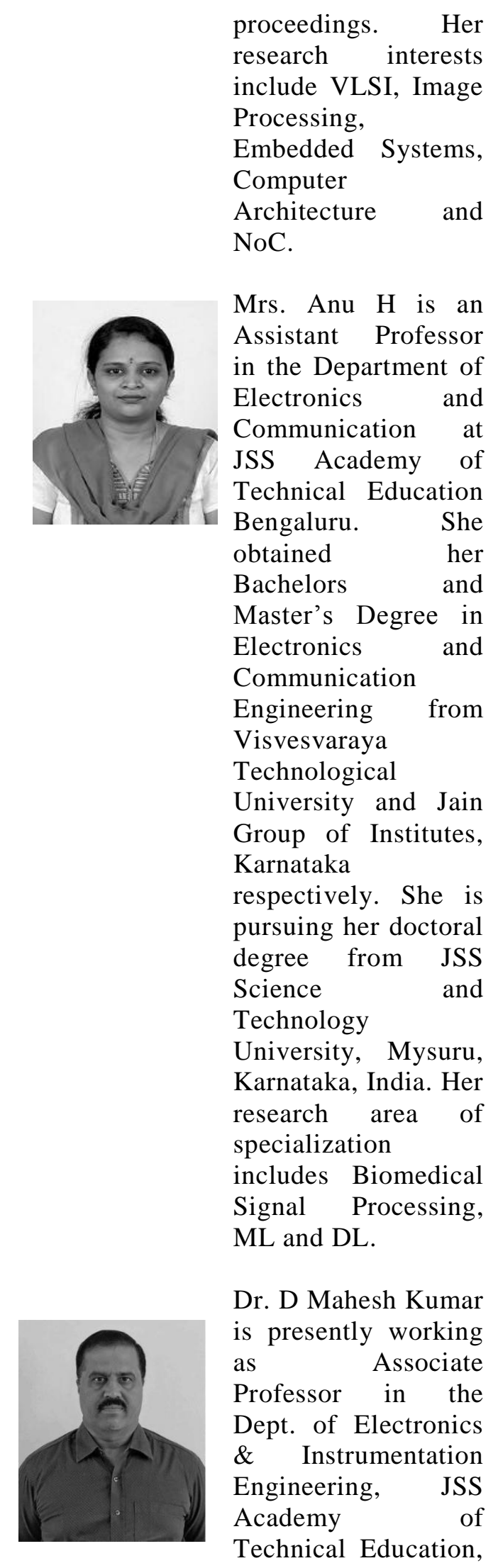

Bengaluru. $\mathrm{He}$ is working in the teaching field from the past 20 years and has published more than 23 papers in International

Journals, National and International Conferences. $\mathrm{He}$ is presently guiding five research scholars under Visvesvaraya Technological University. His research areas include Biomedical Signal Processing, Sensors \& Transducers, Networking etc.

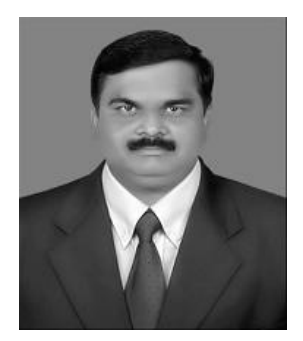

Dr. Aravinda. H.S. received the $\mathrm{BE}$ in Electronics \& Communication

Engg, from Bangalore

University, M.Tech. degree in BMI from Mysore University and Ph.D. in Electronics \& Communication Engineering from Visvesvaraya Technological Technology, Belagavi in the year 2013. He has got a teaching, Research \& administrative experience of nearly 22 years in various engineering colleges in Karnataka. Currently, he is working as Professor in the Dept. of 


Electronics \&
Communication
Engg, JSS Academy
of Technical
Education (JSSATE)
in Bengaluru. He has
published more than
70 research papers in
various National,
International and
Journals is
Conferences in India
$\&$ abroad. He is
guiding 06 research
scholars. He is
currently working on
VGST project on IoT
of Rs 30Lakhs.

